\title{
Colonic granular cell tumor: Report of 11 cases and management with review of the literature
}

\author{
YAHUA CHEN $^{1}$, YANGYANG CHEN ${ }^{2}$, XIAOQIONG CHEN $^{2}$, LIANG CHEN $^{2}$ and WEI LIANG ${ }^{2}$ \\ ${ }^{1}$ Department of Gastroenterology, Affiliated Hospital of Putian University, and Affiliated Putian Hospital of \\ Southern Medical University, Putian, Fujian 351100; ${ }^{2}$ Department of Gastrointestinal Endoscopy, \\ Fujian Provincial Hospital, Teaching Hospital of Fujian Medical University, Fuzhou, Fujian 350001, P.R. China
}

Received August 16, 2016; Accepted January 24, 2018

DOI: $10.3892 / \mathrm{ol} .2018 .8811$

\begin{abstract}
Colonic granular cell tumors (GCTs) are rare and benign, but have malignant potential. The recent progress in endoscopic procedures and technology facilitate the diagnosis and treatment of GCTs. The present study described 11 cases of colonic GCTs diagnosed between March 2010 and April 2015, including patient clinical and histopathological features. Patients were generally asymptomatic. The most common symptoms were hematoquezia and abdominal pain. The male/female ratio was 7:4; age range was 40-67 years. Colonoscopy revealed a yellowish or white, solid and well-circumscribed tumor covered by normal mucosa. Endoscopic ultrasonography (EUS) showed a homogenous, or granular-type heterogeneous, hypoechoic solid tumor originating from the submucosal layer (8 cases) or muscularis mucosae (3 cases), with maximum diameters $0.3-3.0 \mathrm{~cm}$. All patients underwent endoscopic submucosal dissection or endoscopic submucosal excavation without complication. All tumors were block removed. The mean operative time was $38 \mathrm{~min}$ (range, 31-50 min). Immunohistochemical analysis of all specimens confirmed the diagnosis of GCT by positive staining of S-100 protein. No recurrence or metastasis was observed during follow-up periods of 11 months to 5 years. In conclusion, colonoscopy, EUS and immunohistological examination increase the accuracy of diagnosis of GCTs. Endoscopic management is feasible and safe for GCT treatment.
\end{abstract}

\section{Introduction}

Granular cell tumors (GCTs) are rare and were first described by Abrikossof in 1926 (1). These tumors are generally benign

Correspondence to: Dr Wei Liang, Department of Gastrointestinal Endoscopy, Fujian Provincial Hospital, Teaching Hospital of Fujian Medical University, Fuzhou, Fujian 350001, P.R. China E-mail: liangwei8996@163.com

Key words: colonic granular cell tumor, endoscopic ultrasonography, endoscopic submucosal dissection, endoscopic submucosal excavation and only $1-2 \%$ of cases were reported malignant $(2,3)$. The malignant form has a reported 3-year mortality rate of $60 \%$ (4). GCTs have been reported in soft tissue, including skin, tongue, subcutaneous tissue and skeletal muscle. Multiple lesions in the skin occur in $\sim 16 \%$ of all cases (2); however, gastrointestinal involvement, particularly the colon, is extremely rare $(5,6)$. Colonic GCTs are often asymptomatic and may be detected incidentally during colonoscopy screening or through examination performed for non-specific gastrointestinal symptoms.

To date, the majority of what is known regarding GCTs is limited to case reports. For example, Hong and Lim (7) reported a case of GCT in the cecum, which was removed via laparoscopic approach. Cha et al (8) described a case of GCT in the descending colon, which was treated by endoscopic mucosal resection. Due to its rarity, arguments remain regarding diagnosis and treatment. The development of endoscopic technology has provided a new perspective on diagnosis and treatment for colonic GCTs. The present study describes 11 cases of colonic GCTs, including clinical manifestations, endoscopic and endoscopic ultrasonography (EUS) features, pathology, immunohistochemistry, treatment, and morbidity. Special attention is given to the safety and feasibility of endoscopic treatment.

\section{Materials and methods}

Preoperative evaluation. Patients who received a diagnosis of GCT between March 2010 and April 2015 at Gastrointestinal Endoscopy Center of Fujian Provincial Hospital (Fuzhou, China) were included in the present study. The following data were registered: Patient demographics, clinical manifestations, endoscopic characteristics, EUS appearance, pathology, immunohistochemistry, treatment and associated morbidity.

All patients underwent blood tests, including hematic biometry and clotting times, prior to the endoscopic procedure. Colonoscopy, EUS, chest X-ray and abdominal computerized tomography were performed to determine the feasibility of endoscopic treatment. All patients provided signed informed consent to accept the endoscopic procedure after receiving information regarding the treatment, risks, and benefits. Written informed consent was received from all patients for publication of the data in the present study. 
Under EUS, the following were recorded: Tumor location, color, maximum diameter, superficial appearance and the involved layer within the colonic wall. Lesions $\leq 2 \mathrm{~cm}$ in maximum diameter and limited to the mucosa or submucosal layer were selected for endoscopic submucosal dissection (ESD). Lesions in the submucosal layer or $>2 \mathrm{~cm}$ in diameter were selected for endoscopic submucosal excavation (ESE).

Endoscopic resection of GCTs. A single-accessory-channel endoscope with a water-jet (PCF-Q260AZI; Olympus Corporation, Tokyo, Japan) was used. A short transparent cap (ND-201-11802; Olympus Corporation) was used to ensure a clear endoscopic view and apply tension to the connective tissue during the dissection. $\mathrm{CO}_{2}$ insufflation was employed to alleviate abdominal discomfort during the ESD procedure. Several types of electrosurgical knives were applied depending on necessity, including the dual, hook or insulated-tip (KD-650Q, KD-260R and KD611, respectively; Olympus Corporation). The HybridKnife system (ERBE, Tübingen, Germany) was used as the electrosurgical generator. Injection needles (NM4L1), argon plasma coagulation unit (APC300, ERBE), snares, hot biopsy forceps and clips (SD230U20, FD410LR and HX610-135, respectively; Olympus Corporation) were used during the operation.

The standard ESD procedure has been previously described by numerous authors (9) (Fig. 1). Herein, the specific protocol used in the submucosal injection step was highlighted. Due to the duration required for ESD, a solution consisting of $10 \%$ sodium hyaluronate, $5 \%$ lidocaine and $0.5 \%$ indicarmine dye was injected into the submucosa around the lesion, helping to lift the mucosa, and reduce the risk of perforation.

One patient with a tumor $3 \mathrm{~cm}$ in diameter received ESE treatment (Fig. 2), as follows. To lift the tumor, a submucosal injection of several milliliters of the mixed solution aforementioned around the lesion was performed using a 23-gauge disposable needle. A mucosectomy was performed in which the superficial mucosa was incised using a dual knife. The tumor was carefully resected from the connecting tissue using a proper knife (dual or insulation-tipped) to achieve an en bloc resection. For closure, exposed vessels on the surface or at the edge of the wound were coagulated with hot biopsy forceps to prevent delayed bleeding and metallic clips were used to close the wound. The duration between marking foci and complete resection of the tumor was noted.

Pathological diagnosis. All resected specimens were fixed in $10 \%$ formalin solution for $6-8 \mathrm{~h}$ at room temperature. Thereafter, they were embedded in paraffin and cut into $5-\mu \mathrm{m}$ sections. Hematoxylin-eosin staining was performed in accordance with a previous study (10). Immunohistochemical examination was conducted for suspected GCTs. For immunohistochemical analysis, paraffin-embedded sections were deparaffinized in xylene for $5 \mathrm{~min}$ and hydrated in graded ethanol (100\% ethanol for $5 \mathrm{~min}, 95 \%$ ethanol for $3 \mathrm{~min}$, $85 \%$ ethanol for $3 \mathrm{~min}, 80 \%$ ethanol for $3 \mathrm{~min}$ and $75 \%$ ethanol for $3 \mathrm{~min}$, respectively) to prior to immunostaining. Sections were soaked in $10 \mathrm{mM}$ citrate buffer ( $\mathrm{pH}$ 6.0) and treated at $125^{\circ} \mathrm{C}$ for 5 min using a pressure boiler (Decloaking chamber, Biocare Medical LCC, Pacheco, CA, USA) to perform heat-induced antigen retrieval. Sections were left in the pressure boiler to cool following complete boiling. Specimens were then immersed in $5 \%$ bovine serum albumin solution at $37^{\circ} \mathrm{C}$ for 40 mins to block non-specific interaction sites. Sections were incubated with rabbit anti-human S100 (1:6,000; cat. no. PL032696R; Dako; Agilent Technologies, Inc., Santa Clara, CA, USA) overnight at $4^{\circ} \mathrm{C}$, and then with goat anti-rabbit biotin-conjugated secondary antibody (1:2,000; cat. no. A21 220; Abcam, Cambridge, UK) at $37^{\circ} \mathrm{C}$ for $30 \mathrm{~min}$, and subsequently washed three times with PBS. Staining was performed at room temperature for $30 \mathrm{~min}$, using the avidin-biotin-peroxidase complex method (Vectastain; Vector Laboratories, Inc., Burlingame, CA, USA). Sections were counterstained with hematoxylin-eosin for $1 \mathrm{~min}$ at room temperature. Following staining, sections were dehydrated, mounted and viewed via light microscopy (original magnification, $\mathrm{x} 200)$.

Follow-up protocol. Patients with lesions $<1 \mathrm{~cm}$ and benign tumors determined by pathology were followed-up annually. Patients with tumors $>1 \mathrm{~cm}$ or considered histopathologically atypical were supervised every 6 months for the first 2 years and annually thereafter. All patients were monitored for recurrence using thoracic computed tomography scans, endoscopy and EUS.

\section{Results}

Eleven patients ( 7 males and 4 females) with a mean age of 49.91 years (range, 40-67 years) were diagnosed with GCT (Table I). Eight patients were asymptomatic and the tumors were identified via colonoscopy screening. Three patients were referred to the Gastrointestinal Endoscopy Center of Fujian Provincial Hospital complaining of hematochezia (1 patient) and abdominal pain (2 patients). Eight lesions were located in the ascending colon and 3 in the cecum; all of them were solitary.

White-light colonoscopy revealed a yellowish or white, solid and well-circumscribed tumor covered by smooth superficial mucosa (Fig. 3A and B). On EUS, these colonic GCTs appeared as homogenous originating from the muscularis mucosa (3 cases; Fig. 3C) or a granular-type heterogeneous hypoechoic solid tumor arising from the submucosal layer ( 8 cases; Fig. 3D). The tumors were well circumscribed with no compression of neighboring organs and no lymph node was affected. The tumor diameters ranged between 0.3 and $3.0 \mathrm{~cm}$, with a mean diameter of $1.1 \mathrm{~cm}$.

All patients underwent endoscopic resection, based on the tumor characteristics. Ten patients with tumor size $\leq 2 \mathrm{~cm}$ and located within the muscularis mucosa or submucosal layer underwent standardized ESD to insure complete resection of the tumor. Another patient with a GCT $3 \mathrm{~cm}$ in diameter and originating from the submucosal layer accepted ESE for treatment. Tumors were removed en bloc without complications. The mean operative duration was $38 \mathrm{~min}$ (range, 31 to $50 \mathrm{~min}$ ).

Pathological diagnosis of the tumor was performed on all patients. No GCT tissue was detected at the bottom or on the edge of the specimens, indicating complete resection. Histologically, all GCT lesions showed a well-circumscribed 

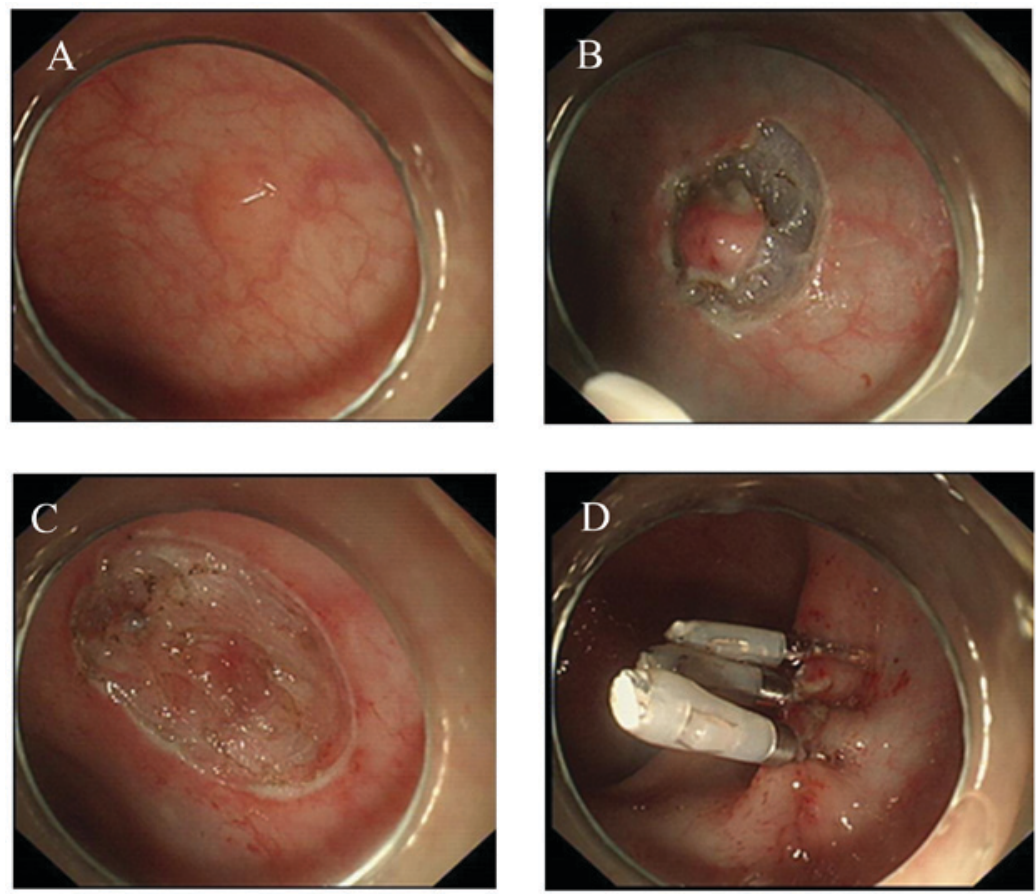

Figure 1. ESD procedure. (A) Submucosal injection of mixed solution around the lesion to lift the tumor. (B) Submucosal dissection of the lesion. (C) Wound appearance. (D) Wound closing using clips. ESD, endoscopic submucosal dissection.
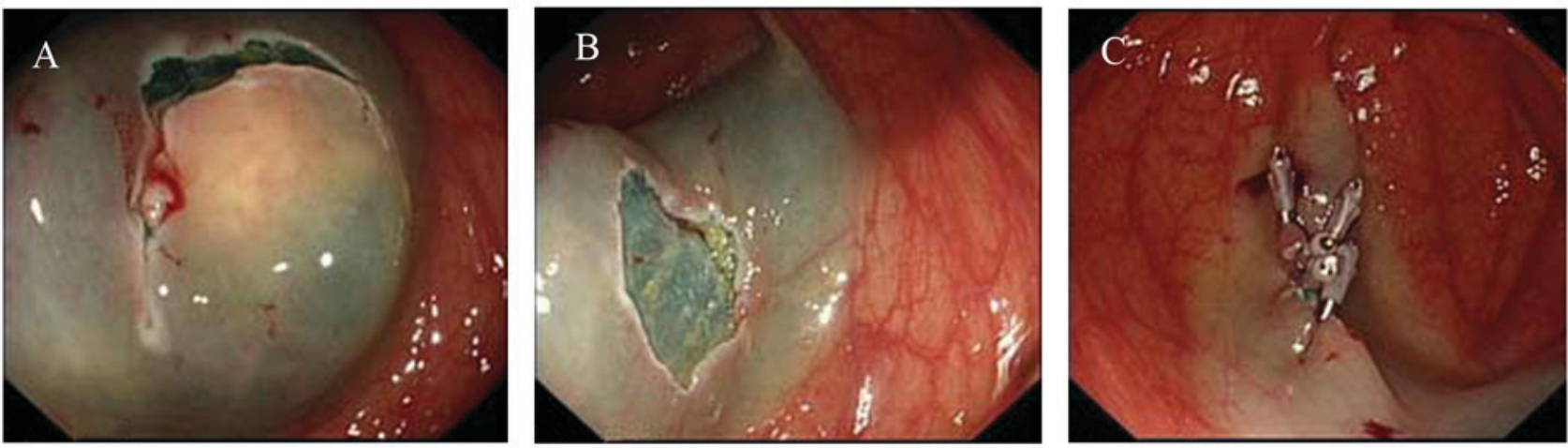

Figure 2. ESE procedure. (A) Submucosal injection of mixed solution and mucosectomy. (B) Wound surface. (C) Wound closure with clips. ESE, endoscopic submucosal excavation.

Table I. Patient clinical characteristics.

\begin{tabular}{ccllllrr}
\hline Patient & Age, years & Sex & Presentation & Tumor location & Invading layer & Tumor size, $\mathrm{cm}^{\mathrm{a}}$ & Excision \\
\hline 1 & 60 & Male & Asymptomatic & Ascending colon & Submucosa & 0.6 & ESD \\
2 & 67 & Female & Asymptomatic & Cecum & Submucosa & 0.5 & ESD \\
3 & 42 & Male & Hematochezia & Ascending colon & Submucosa & 3.0 & ESE \\
4 & 50 & Male & Asymptomatic & Ascending colon & Muscularis mucosa & 0.4 & ESD \\
5 & 40 & Female & Abdominal pain & Cecum & Submucosa & 1.2 & ESD \\
6 & 52 & Male & Asymptomatic & Ascending colon & Muscularis mucosa & 0.8 & ESD \\
7 & 47 & Female & Asymptomatic & Ascending colon & Submucosa & 1.5 & ESD \\
8 & 45 & Male & Abdominal pain & Cecum & Submucosa & 1.0 & ESD \\
9 & 57 & Female & Asymptomatic & Ascending colon & Muscularis mucosa & 0.8 & ESD \\
10 & 45 & Male & Asymptomatic & Ascending colon & Submucosa & 1.5 & ESD \\
11 & 44 & Male & Asymptomatic & Ascending colon & Submucosa & 0.8 & ESD \\
\hline
\end{tabular}

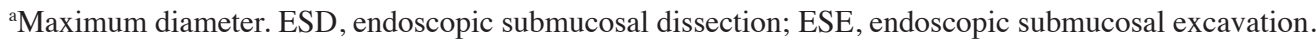



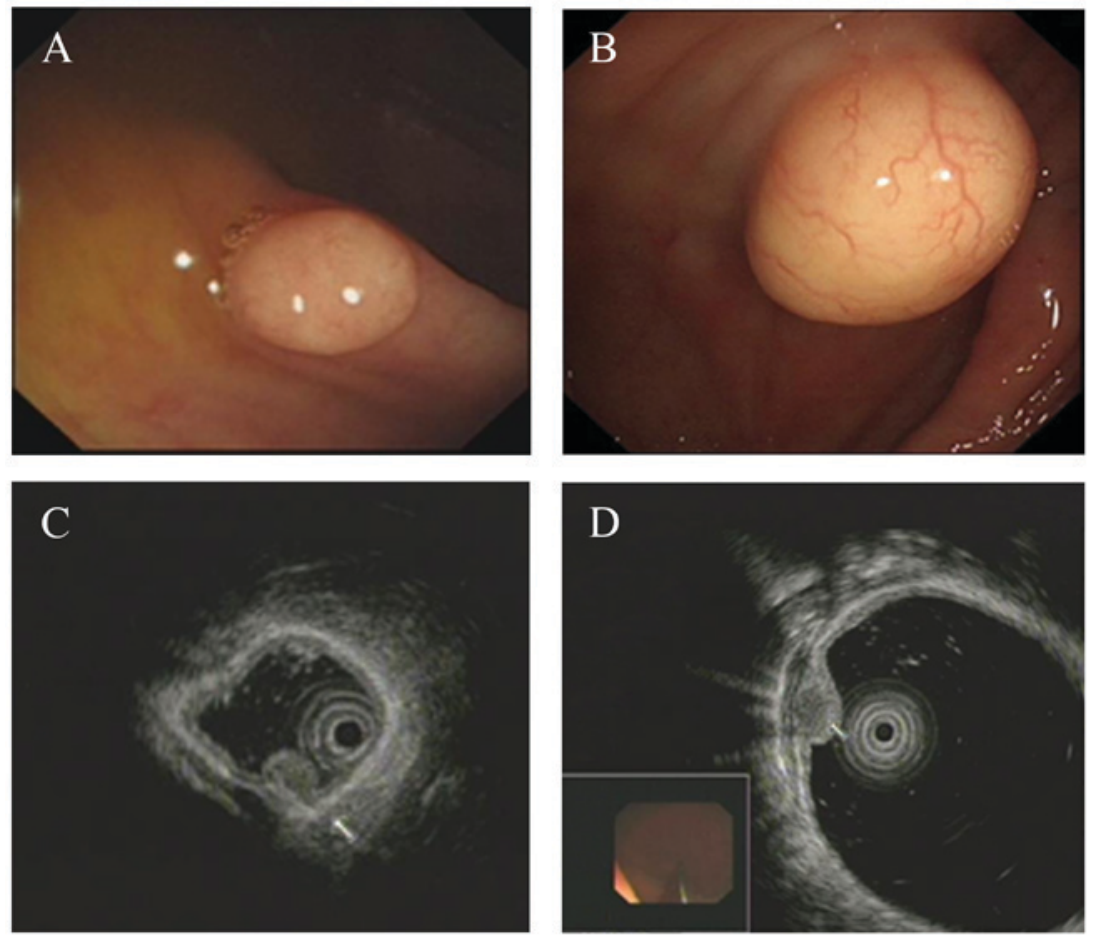

Figure 3. Colonoscopy and EUS image of colonic GCTs. (A) Colonoscopic view of a 0.4-cm GCT located in the ascending colon. (B) Colonoscopic view of a $3.0-\mathrm{cm}$ GCT located in the cecum. (C) Homogenous and hypoechoic lesion originating from muscularis mucosa, by EUS. (D) GCT image by EUS, showing granular-type heterogeneous and hypoechoic lesion confined to the submucosal layer. EUS, endoscopic ultrasonography; GCTs, granular cell tumors.
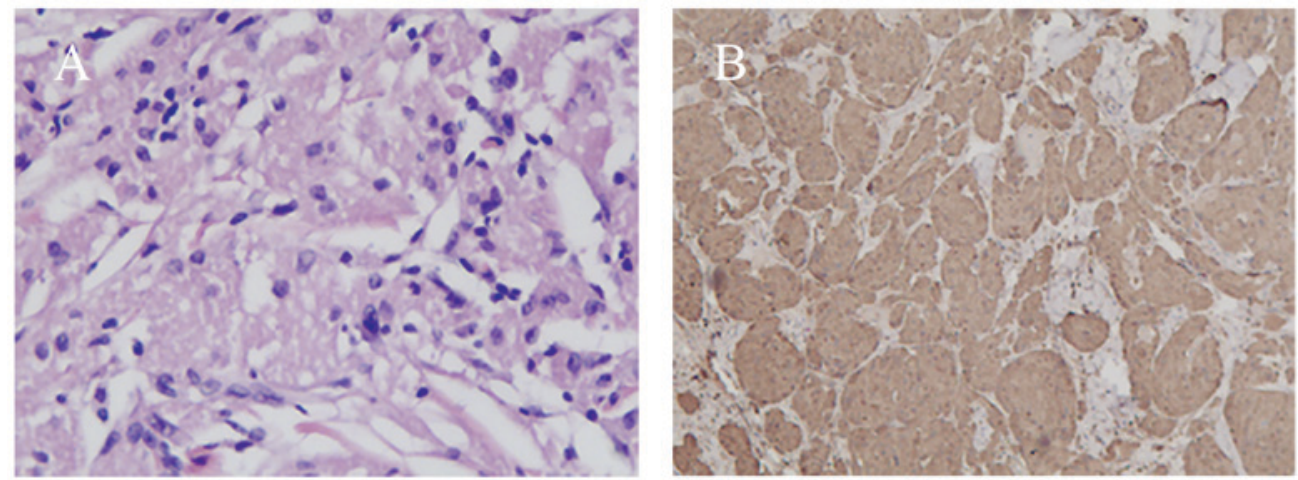

Figure 4. Histologic features of colonic granular cell tumor. (A) Hematoxylin and eosin showing ribbons of round-to-polyhedral cells that were characterized by small, uniform nuclei surrounded by abundant granular eosinophilic cytoplasm (original magnification, x200). (B) Immunohistochemical analysis revealed positive staining of S-100 in the nucleus and cytoplasm (original magnification, x200).

growth pattern within the submucosal layer. The gross specimen was a solitary and peanut-like nodule with a yellowish cross-section. Microscopically, the neoplastic cells were characterized by small, uniform nuclei surrounded by abundant granular eosinophilic cytoplasm (Fig. 4A). Typical histopathological features confirmed the diagnosis of GCT. Further immunohistochemical analysis revealed positive staining for S-100 in the nucleus and cytoplasm for all cases (Fig. 4B). None of the lesions met the criteria for malignant or atypical GCT (3). There, all were determined to be histologically benign.

All patients completed follow-ups of 11 months to 5 years. None of the patients complained of any discomfort or experienced massive weight loss during their follow-up period. Colonoscopy and EUS were performed in all patients and none exhibited recurrence or metastasis.

\section{Discussion}

GCTs are a rare type of soft tissue tumors that may be detected anywhere throughout the body, most commonly in the oral cavity, skin, skeletal muscle and subcutaneous tissue, but also in the nervous system, respiratory tract, and female genital organs $(11,12)$. GCTs are relatively uncommon in the gastrointestinal track, accounting for $5-11 \%$ of all GCTs $(5,6,13)$. To date, $\sim 130$ cases of colonic GCTs have been reported in the English literature (14). Among the relatively rare locations, the esophagus is involved most frequently, then the duodenum, anus and stomach. Least common is the colon and rectum, where primarily the ascending colon, cecum, appendix, and rectum are affected (8). In the present report, the tumors were identified in the ascending colon ( 8 cases) and cecum ( 3 cases). 
The sex and age distribution of GCT remains controversial in the literature, partially due to cases that were insufficiently described. Singhi et al (14) reported an equal sex distribution, with ages ranging between 31 and 60 years. An et al (15) observed a male predominance, with a wider age distribution between 21 and 75 years. In the present study, the male-to-female ratio was 7:4, with ages ranging between 40 and 67 years.

Colonic GCTs tend to be asymptomatic and usually follow a benign course. The lesions are usually identified during colonoscopy screening or examinations performed for other reasons. Patients who had discomfort may exhibit larger lesions. However, this association is not strong regarding colonic GCTs. Common symptoms are non-specific, including abdominal discomfort, hematochezia and changes in bowel habits. In the present study, 8 patients were asymptomatic and 3 complained of hematochezia (1 patient) and abdominal pain (2 patients). An association between the symptoms of the 3 patients and colonic GCT of the mucosal layer is unlikely. Multiple GCTs in children may be indicative of neurofibromatosis (16), Noonan syndrome (17), growth retardation or Hodgkin's disease in remission (18). However, the statistical power is not sufficient due to the scarcity of cases.

In the present cases, the white-light colonoscopy showed a yellowish or white, well-circumscribed tumor covered by normal mucosa. A few cases may show rough mucosa or ulceration on the top of the lesion, due to an inflammatory reaction to the mucosa. The lesion is usually solitary and $<2 \mathrm{~cm}$ in diameter. However, multiple lesions within the gastrointestinal track or involved extra-gastrointestinal sites have also been reported in 10-20\% of all GCTs (19), and the largest tumor has been $4 \mathrm{~cm}$ in diameter (20).

EUS is essential to determine the invasion depth and nature of GCTs. Typical colonic GCTs are characterized by homogeneous or mild heterogeneous hypoechoic nodules with a growth pattern within the mucosa or submucosa. In the current study, 8 tumors were detected in the submucosal layer with a hypoechoic but granular-type heterogeneous ultrasonography image. This particular feature may facilitate the diagnosis of colonic GCTs. However, colonoscopy examination or EUS features are not reliable enough to confirm a diagnosis of GCT.

The final diagnosis of GCT is based on histopathology findings. The typical pathological characteristics of GCT and positive staining for S-100 protein support an accurate diagnosis; the key is to get precise GCT tissue. GCTs located at the mucosa may benefit from biopsy. However, for those restricted to the submucosal area an endoscopic forceps biopsy is not recommended, because it may not pick up the actual lesion, but destroy the tumor integrity with subsequent bleeding.

EUS-guided fine needle aspiration is valuable for pathological determination. However, the extracted biopsy tissue is of limited size and the malignant location may be missed, leading to a false diagnosis. Complete resection is the optimal strategy to obtain specimens of submucosal tumors. None of the cases in the present report underwent biopsy, considering that all lesions were submucosal tumors.

GCTs are generally considered benign with malignant potential (1-2\%) (19,21). For benign cases, endoscopic mucosal resection or ESD is the best strategy for tumors $<2 \mathrm{~cm}$ in diameter $(22,23)$. Previously, partial colectomy was advised for tumors $>2 \mathrm{~cm}$ in diameter (24). However, in our experience, lesions measuring 3-5 $\mathrm{cm}$ and limited to the submucosa may be completely resected by ESE, without bleeding or perforation. This requires a thorough hemostatic procedure at the edge and bottom of the wound, and excavation should be performed close to the submucosal layer while incising the bottom of the tumor.

At present, perforation is the greatest concern of endoscopists (25). The development of endoscopic techniques provides several closure approaches during ESD to deal with perforation, including the following: Clipping; clipping and then strengthening with an endoloop; purse string suture with metallic clips and endoloop; and interrupted suture with endoloop and metallic clip (26). The advent of endoscopic closure techniques has expanded the indications for ESD for colonic GCTs located within the submucosal layer, avoiding invasive procedures.

The first malignant GCT was described by Ravich in 1945 (27). Notably, this particular clinically malignant tumor may be histologically malignant or benign. The histological features of malignant GCTs have been described previously $(3,11)$. Herein, the clinical features that may indicate a GCT with malignant potential, all of which are high-risk signals that call for clinicians' special attention are summarized as follows: Advanced patient age, tumor size $>5 \mathrm{~cm}$, rapid recent growth, and an infiltrative growth pattern. It is recommended that patients with suspected GCTs undergo a preoperative examination that includes EUS and abdominal computerized tomography, to determine the possibility of endoscopic treatment. Traditional surgery is indicated for a GCT measuring $5 \mathrm{~cm}$ in maximum diameter, an infiltrative growth pattern invading the muscular layer and lympho-vascular invasion, or further metastasis. The role of chemotherapy and radiotherapy remains indefinite, as there are only a few cases of malignant GCTs that responded to chemotherapy $(28,29)$.

In summary, colonic GCTs are rare, primarily occur in the right colon and typically follow a benign course. The initial clinical manifestation and endoscopic appearance is non-specific, and therefore a correct diagnosis is difficult to achieve. The advancement of endoscopic technique has partially replaced partial colectomy for the treatment of benign GCTs. However, traditional surgery remains the optimal strategy for malignant cases.

\section{Acknowledgements}

Not applicable.

\section{Funding}

No funding was received.

\section{Availability of data and materials}

The datasets used and/or analyzed during the current study are available from the corresponding author on reasonable request.

\section{Authors' contributions}

YYC conceived and designed the study. XC and LC collected all of the data. YHC and WL analyzed the data, drafted the article and made critical revisions to the article. All of the authors gave final approval of the version to be published. 


\section{Ethics approval and consent to participate}

All patients provided written informed consent to accept the endoscopic procedure, after receiving information regarding the treatment, risks and benefits.

\section{Consent for publication}

Written informed consent was received from all patients for publication of the data in the present study.

\section{Competing interests}

The authors declare no competing interests.

\section{References}

1. Abrikossoff AI: Weitere untersuchungen über myoblastenmyome Virchows Archiv Für Pathologische Anatomie Und Physiologie Und Für Klinische Medicin 280: 723-740, 1931.

2. Khansur T, Balducci L and Tavassoli M: Granular cell tumor. Clinical spectrum of the benign and malignant entity. Cancer 60: 220-222, 1987.

3. Fanburg-Smith JC, Meis-Kindblom JM, Fante R and Kindblom LG: Malignant granular cell tumor of soft tissue: Diagnostic criteria and clinicopathologic correlation. Am J Surg Pathol 22: 779-794, 1998.

4. Akahane K, Kato K, Ogiso S, Sakaguchi K, Hashimoto M, Ishikawa A, Kato T, Fuwa Y, Takahashi A and Kobayashi K: Malignant granular cell tumor of the breast: Case report and literature review. Breast Cancer 22: 317-323, 2015.

5. Lack EE, Worsham GF, Callihan MD, Crawford BE, Klappenbach S, Rowden G and Chun B: Granular cell tumor: A clinicopathologic study of 110 patients. J Surg Oncol 13: 301-316, 1980.

6. Johnston J and Helwig EB: Granular cell tumors of the gastrointestinal tract and perianal region: A study of 74 cases. Dig Dis Sci 26: 807-816, 1981

7. Hong R and Lim SC: Granular cell tumor of the cecum with extensive hyalinization and calcification: A case report. World $\mathrm{J}$ Gastroenterol 15: 3315-3318, 2009.

8. Cha JM, Lee JI, Joo KR, Choe JW, Jung SW, Shin HP and Lim SJ: Granular cell tumor of the descending colon treated by endoscopic mucosal resection: A case report and review of the literature. J Korean Med Sci 24: 337-341, 2009.

9. Take I, Shi Q, Qi ZP, Cai SL, Yao LQ, Zhou PH and Zhong YS: Endoscopic resection of colorectal granular cell tumors. World $\mathrm{J}$ Gastroenterol 21: 13542-13547, 2015.

10. Feldman AT and Wolfe D: Tissue processing and hematoxylin and eosin staining. Methods Mol Biol 1180: 31-43, 2014.

11. Ensari A: Granular cell tumor: What's new in diagnosis and treatment? Turk J Gastroenterol 18: 135-138, 2007.

12. Haikal F, Maceira J, Dias E and Ramos-E-Silva M: Histogenesis of Abrikossoff tumour of the oral cavity. Int J Dent Hyg 8: 53-62, 2010.

13. Parfitt JR, McLean CA, Joseph MG, Streutker CJ, Al-Haddad S and Driman DK: Granular cell tumours of the gastrointestinal tract: Expression of nestin and clinicopathological evaluation of 11 patients. Histopathology 48: 424-430, 2006.
14. Singhi AD and Montgomery EA: Colorectal granular cell tumor: A clinicopathologic study of 26 cases. Am J Surg Pathol 34: 1186-1192, 2010

15. An S, Jang J, Min K, Kim MS, Park H, Park YS, Kim J, Lee JH, Song HJ, Kim KJ, et al: Granular cell tumor of the gastrointestinal tract: Histologic and immunohistochemical analysis of 98 cases. Hum Pathol 46: 813-819, 2015.

16. Sahn EE, Dunlavey ES and Parsons JL: Multiple cutaneous granular cell tumors in a child with possible neurofibromatosis. J Am Acad Dermatol 36: 327-330, 1997.

17. Moos D, Droitcourt C, Rancherevince D, Marec Berard P and Skowron F: Atypical granular cell tumor occurring in an individual with Noonan syndrome treated with growth hormone. Pediatr Dermatol 29: 665-666, 2012.

18. De Raeve L, Roseeuw D and Otten J: Multiple cutaneous granular cell tumors in a child in remission for Hodgkin's disease. J Am Acad Dermatol 47 (Suppl 2): S180-S182, 2002.

19. Saleh H, El-Fakharany M and Frankle M: Multiple synchronous granular cell tumors involving the colon, appendix and mesentery: A case report and review of the literature. J Gastrointestin Liver Dis 18: 475-478, 2009.

20. Choi SM, Hong SG, Kang SM, Chae BG, Kim SJ, Park PK and Park HS: A case of malignant granular cell tumor in the sigmoid colon. Clin Endosc 47: 197-200, 2014.

21. Thacker MM, Humble SD, Mounasamy V, Temple HT and Scully SP: Case report. Granular cell tumors of extremities: Comparison of benign and malignant variants. Clin Orthop Relat Res 455: 267-273, 2007.

22. Hoteya S, Yahagi N, Iizuka T, Kikuchi D, Kawano K, Noguchi T, Mizuno $\mathrm{H}$ and Hashimoto M: Endoscopic resection for early gastric cancers by EMR/ESD. Gan To Kagaku Ryoho 34: 16-20, 2007 (In Japanese).

23. Goto O, Uraoka T, Horii J and Yahagi N: Expanding indications for ESD: Submucosal disease (SMT/carcinoid tumors). Gastrointest Endosc Clin N Am 24: 169-181, 2014.

24. Chen J, Wang L, Xu J, Pan T, Shen J, Hu W and Yuan X: Malignant granular cell tumor with breast metastasis: A case report and review of the literature. Oncol Lett 4: 63-66, 2012.

25. Ma MX and Bourke MJ: Complications of endoscopic polypectomy, endoscopic mucosal resection and endoscopic submucosal dissection in the colon. Best Pract Res Clin Gastroenterol 30: 749-767, 2016.

26. Shi Q, Chen T, Zhong YS, Zhou PH, Ren Z, Xu MD and Yao LQ: Complete closure of large gastric defects after endoscopic full-thickness resection, using endoloop and metallic clip interrupted suture. Endoscopy 45: 329-334, 2013.

27. Ravich A, Stout AP and Ravich RA: Malignant granular cell myoblastoma involving the urinary bladder. Ann Surg 121: 361-372, 1945.

28. Chiang MJ, Fang TJ, Li HY, Chen IH and Lee KF: Malignant granular cell tumor in larynx mimicking laryngeal carcinoma. Am J Otolaryngol 25: 270-273, 2004.

29. Morita S, Hiramatsu M, Sugishita M, Gyawali B, Shibata T, Shimokata T, Urakawa H, Mitsuma A, Moritani S, Kubota T, et al: Pazopanib monotherapy in a patient with a malignant granular cell tumor originating from the right orbit: A case report. Oncol Lett 10: 972-974, 2015.

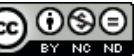

This work is licensed under a Creative Commons Attribution-NonCommercial-NoDerivatives 4.0 International (CC BY-NC-ND 4.0) License. 